\title{
Freeform design of a two-reflector system to collimate and shape a point source distribution
}

\author{
A.H. van Roosmalen ${ }^{1, *}$, M.J.H. Anthonissen ${ }^{1}$, W.L. IJzerman ${ }^{1,2}$, and J.H.M. ten Thije Boonkkamp ${ }^{1}$ \\ ${ }^{1}$ CASA, Department of Mathematics and Computer Science, Eindhoven University of Technology, \\ PO Box 513, 5600 MB Eindhoven, The Netherlands \\ ${ }^{2}$ Signify Research, High Tech Campus 7, 5656 AE Eindhoven, The Netherlands
}

\begin{abstract}
We present a method to design a freeform two-reflector system to collimate and shape a beam from a point source. An important generalization compared to previous research is that the output beam can be in an arbitrary direction. The design problem is based on a generalized Monge-Ampère equation. This equation is solved using a least-squares algorithm for non-quadratic cost functions. We test our algorithm on two cases, first, uniform source and target distributions, and second, an elliptic Gaussian intensity of a laser diode to a ring-shaped illuminance. We are able to obtain good solutions in both cases.
\end{abstract}

\section{Introduction}

In this article we will give a summary of our paper in Optics Express [1]. We consider the design of an optical system with two freeform reflector surfaces for collimating and shaping the distribution from a point source. This is regularly linked to beam shaping for lasers. The source of a laser is often a laser diode, which can be modeled as a point source. In general, its output is collimated with a lens and subsequently shaped. Combining these two functions into one optical system of two reflector surfaces reduces the number of optical surfaces needed. To achieve this, we adapt a method developed by Prins [2], Yadav [3], Romijn [4] et al. for other optical systems.

\section{Mathematical model}

Our goal is to construct an optical map $\boldsymbol{m}$ from the source domain $\mathcal{S}$ to the target domain $\mathcal{T}$ such that the source density $f$ is mapped to a target density $g$. The set $\mathcal{S}$ is a subset of the unit sphere and $\mathcal{T}$ contains two-dimensional position vectors on the target plane, which is at a distance $l$ from the point source. This target plane is perpendicular to the direction of the outgoing beam, given by the vector $\hat{\boldsymbol{a}}_{3}$. This vector can be anywhere on the unit sphere, which gives freedom in designing optical systems. We regard $\hat{\boldsymbol{a}}_{3}$ as a rotation from the $z$-axis with a rotation matrix $\boldsymbol{A}$, defined by the polar angle $\varphi$ and azimuthal angle $\theta$, so $\hat{\boldsymbol{a}}_{3}=\boldsymbol{A} \hat{\boldsymbol{e}}_{3}$. For $\hat{\boldsymbol{s}} \in \mathcal{S}$, the first reflector $\mathcal{R}_{1}$ is defined by $\boldsymbol{r}_{1}(\hat{\boldsymbol{s}})=u(\hat{\boldsymbol{s}}) \hat{\boldsymbol{s}}$, where $u$ is the distance from the point source to the reflector. Similarly, for $\boldsymbol{y} \in \mathcal{T}$, the second reflector $\mathcal{R}_{2}$ is located at a distance $w=w(\boldsymbol{y})$ from the target plane. The reflector is then defined by $\boldsymbol{r}_{2}=\boldsymbol{A}\left(y_{1}, y_{2}, l-w(\boldsymbol{y})\right)^{\top}$. The distance between the reflectors measured along a ray is denoted by $d$. See Fig. 1 for more details. The optical

*e-mail: a.h.v.roosmalen@tue.nl

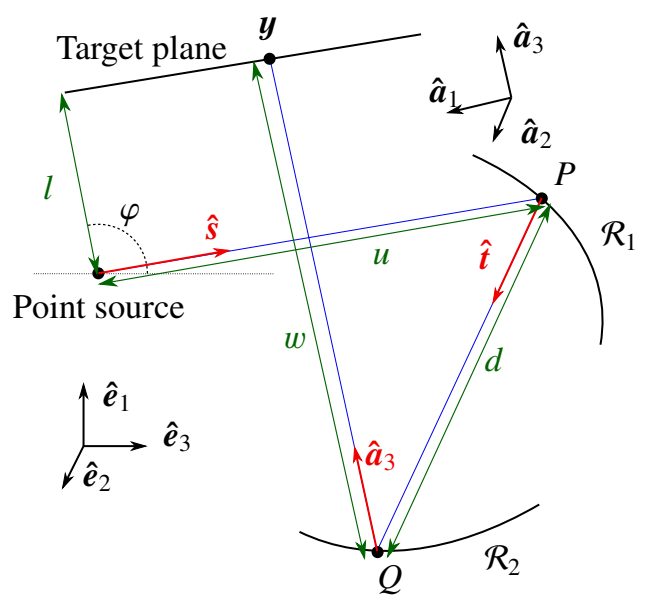

Figure 1: A cross-section of the optical system in the $x, z-$ plane.

path length, $L$, is given by

$$
L=u(\hat{\boldsymbol{s}})+d+w(\boldsymbol{y})
$$

and is constant in $\hat{\boldsymbol{s}}$ and $\boldsymbol{y}$ as a consequence of the MalusDupin theorem [5, Sec. 4.4.3]. Using that $d$ is the distance between the reflectors, i.e., $d=\left|\boldsymbol{r}_{2}-\boldsymbol{r}_{1}\right|$, we can express it as a function of $u$ and $w$. We parameterize the unit direction vectors $\hat{\boldsymbol{s}}$ by the stereographic projection $\boldsymbol{x}$, given by

$$
\boldsymbol{x}=\frac{1}{1+s_{3}}\left(\begin{array}{l}
s_{1} \\
s_{2}
\end{array}\right)
$$

and introduce $\mathcal{X}=\boldsymbol{x}(\mathcal{S})$. We use this to derive an equation of the form

$$
u_{1}(\boldsymbol{x})+u_{2}(\boldsymbol{y})=c(\boldsymbol{x}, \boldsymbol{y}) .
$$


The functions $u_{1}$ and $u_{2}$ are related to the first and second reflector, respectively, given by

$$
\begin{aligned}
& u_{1}=\log \left(\frac{\beta}{u}+\hat{\boldsymbol{s}} \cdot \hat{\boldsymbol{a}}_{3}-1\right), \\
& \left.u_{2}=\log \left(-\frac{w}{\beta}-\frac{1}{2 \beta^{2}}\left[|\boldsymbol{y}|^{2}-L^{2}+l^{2}\right)\right]\right),
\end{aligned}
$$

while $c$ is called the cost function. It is given by

$$
\begin{aligned}
c(\boldsymbol{x}, \boldsymbol{y}) & =\log \left[-\frac{1}{\beta} \hat{\boldsymbol{s}}(\boldsymbol{x}) \cdot \boldsymbol{A}_{2} \boldsymbol{y}+\frac{1-\hat{\boldsymbol{s}}(\boldsymbol{x}) \cdot \hat{\boldsymbol{a}}_{3}}{2 \beta^{2}}|\boldsymbol{y}|^{2}\right. \\
& \left.+\frac{1}{2}\left(\hat{\boldsymbol{s}}(\boldsymbol{x}) \cdot \hat{\boldsymbol{a}}_{3}+1\right)\right],
\end{aligned}
$$

where $\beta=L-l$ is the reduced optical path length and $\boldsymbol{A}_{2}$ is the matrix containing the first two columns of $\boldsymbol{A}$.

Eq. (3) has many possible solutions. This gives the possibility to choose a $c$-concave (or $c$-convex) pair $u_{1}, u_{2}$ as a solution. This means, for $c$-concave, that

$$
\begin{aligned}
& u_{1}(\boldsymbol{x})=\min _{\boldsymbol{y} \in \mathcal{T}}\left(c(\boldsymbol{x}, \boldsymbol{y})-u_{2}(\boldsymbol{y})\right), \\
& u_{2}(\boldsymbol{x})=\min _{\boldsymbol{x} \in \mathcal{X}}\left(c(\boldsymbol{x}, \boldsymbol{y})-u_{1}(\boldsymbol{x})\right) .
\end{aligned}
$$

Alternatively, for a $c$-convex pair, there is a maximum instead of the minimum. As a result, $u_{2}$ is at a stationary point in $\boldsymbol{x}$ and we require

$$
\nabla_{x} c(\boldsymbol{x}, \boldsymbol{y})-\nabla u_{1}(\boldsymbol{x})=\mathbf{0} .
$$

From this, we can in principle compute a mapping $\boldsymbol{m}(\boldsymbol{x})=$ $\boldsymbol{y}$, however, we do not. Instead, we substitute the relation $\boldsymbol{y}=\boldsymbol{m}(\boldsymbol{x})$ into Eq. (7) and subsequently differentiate w.r.t. $x$ to obtain the condition

$$
\boldsymbol{C D} \boldsymbol{m}=\mathrm{D}^{2} u_{1}-\mathrm{D}_{x \boldsymbol{x}} c=: \boldsymbol{P},
$$

where $\mathrm{D} \boldsymbol{m}$ is the Jacobian matrix of $\boldsymbol{m}$ and $\boldsymbol{C}=\mathrm{D}_{x y} c=$ $\left(\frac{\partial^{2} c}{\partial x_{i} \partial y_{j}}\right)$, the mixed derivative matrix, which is a function of $\boldsymbol{x}$ and $\boldsymbol{m}(\boldsymbol{x})$. The matrix $-\boldsymbol{P}$ is equal to the Hessian of $c(., \boldsymbol{y})-u_{1}$. Therefore, if we choose $u_{1}, u_{2}$ as a $c$-concave or $c$-convex pair, we need $\boldsymbol{P}$ to be symmetric negative definite (SND) or symmetric positive definite (SPD), respectively.

The map should also satisfy local energy conservation. For any subset $\tilde{\mathcal{A}} \subset \mathcal{S}$ there is a corresponding set $\mathcal{A}$ of stereographic coordinates. The energy conservation is given by

$$
\iint_{\tilde{\mathcal{A}}} f(\hat{\boldsymbol{s}}) \mathrm{d} S(\hat{\boldsymbol{s}})=\iint_{\boldsymbol{m}(\mathcal{A})} g(\boldsymbol{y}) \mathrm{d} A(\boldsymbol{y}) .
$$

This says that energy should be conserved through the optical system. The energy contained in a part of the source should be equal to that of the corresponding part on the target. When $\tilde{\mathcal{A}}=\mathcal{S}$, we have global energy conservation, meaning that the total flux of the source and target should be equal. Using integration by substitution, we write Eq. (9) in differential form as

$$
\operatorname{det}(\operatorname{Dm})=J(x) \frac{f(\boldsymbol{x})}{g(\boldsymbol{m}(\boldsymbol{x}))}=: F(\boldsymbol{x}, \boldsymbol{m}(\boldsymbol{x})),
$$

where $J$ is the Jacobian resulting from coordinate transformation of the stereographic projection $\hat{s} \rightarrow \boldsymbol{x}$, given by

$$
J(\boldsymbol{x})=\frac{4}{\left(|\boldsymbol{x}|^{2}+1\right)^{2}} .
$$

The boundary condition for this problem is given by the transport boundary condition $\boldsymbol{m}(\partial \mathcal{X})=\partial \mathcal{T}$, stating that the boundary of the source should be mapped to the boundary of the target. Consequentially, all energy is mapped from the source to the target.

Summarizing, we can derive a mapping $\boldsymbol{m}$ from Eq. (8) subject to the transport boundary condition, where $\boldsymbol{P}$ satisfies $\operatorname{det}(\boldsymbol{P})=F \operatorname{det}(\boldsymbol{C})$, due to Eq. (10). Subsequently, we calculate $u_{1}$ and $u_{2}$ from Eq. (7) and (3), respectively, and from those we compute the optical surfaces from Eq. (4).

\section{Least-squares solver}

We solve the problem mentioned above iteratively with a least-squares algorithm. A brief explanation will be given here, for more details, see [1]. We enforce the equality in Eq. (8) by minimizing the functional $J_{I}$ defined as

$$
J_{\mathrm{I}}[\boldsymbol{m}, \boldsymbol{P}]=\frac{1}{2} \iint_{X}\|\boldsymbol{C D} \boldsymbol{m}-\boldsymbol{P}\|_{\mathrm{F}}^{2} \mathrm{~d} \boldsymbol{x} .
$$

The norm $\|.\|_{F}$ is the Frobenius norm. To enforce the boundary condition we minimize the difference between the (given) boundary of $\mathcal{T}$ and the mapping of the boundary of the source:

$$
J_{\mathrm{B}}[\boldsymbol{m}, \boldsymbol{b}]=\frac{1}{2} \int_{\partial X}|\boldsymbol{m}-\boldsymbol{b}|^{2} \mathrm{~d} s,
$$

where $\boldsymbol{b}: \partial \mathcal{X} \rightarrow \partial \mathcal{T}$. We combine these two functionals into a weighted average with parameter $\alpha \in[0,1]$ given by

$$
J[\boldsymbol{m}, \boldsymbol{P}, \boldsymbol{b}]=\alpha J_{\mathrm{I}}[\boldsymbol{m}, \boldsymbol{P}]+(1-\alpha) J_{\mathrm{B}}[\boldsymbol{m}, \boldsymbol{b}] .
$$

All of these functionals are defined on the following spaces

$$
\begin{aligned}
\mathcal{P}(\boldsymbol{m}) & =\left\{\boldsymbol{P} \in\left[C^{1}(\mathcal{X})\right]^{2 \times 2} \mid \operatorname{det}(\boldsymbol{P})=F \operatorname{det}(\boldsymbol{C}), \boldsymbol{P} \mathrm{SPD}\right\}, \\
\mathcal{B} & =\left\{\boldsymbol{b} \in\left[C^{1}(\partial \mathcal{X})\right]^{2} \mid \boldsymbol{b}(\boldsymbol{x}) \in \partial \mathcal{T}\right\}, \\
\mathcal{M} & =\left[C^{2}(\mathcal{X})\right]^{2} .
\end{aligned}
$$

In common parlance, $\mathcal{P}$ contains $2 \times 2$ matrices with differentiable entries such that the stated constraints are fulfilled and $\mathcal{B}$ contains vector-valued functions from the source boundary to the target boundary whose components are differentiable. Similarly, $\mathcal{M}$ contains vector-valued functions, that are twice differentiable.

We cover the domain $X$ by a grid with gridpoints $\boldsymbol{x}_{i j}$. The algorithm to find $\boldsymbol{m}$ is initialized by a guess $\boldsymbol{m}^{0}$ for the mapping. With this mapping we compute the matrix $\boldsymbol{C}^{0}=\boldsymbol{C}\left(\boldsymbol{x}, \boldsymbol{m}^{0}\right)$. Then, we iteratively perform the next steps, either for a fixed number of iterations or until a stop- 


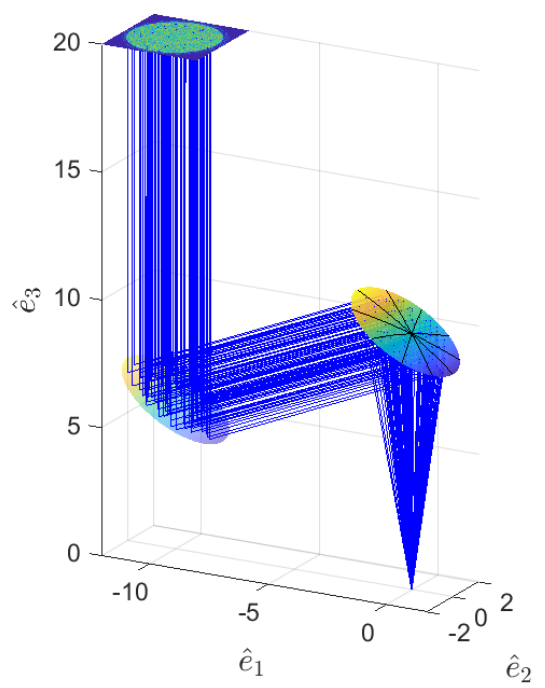

(a) $\varphi=0, \beta=15, u_{0}=10$.

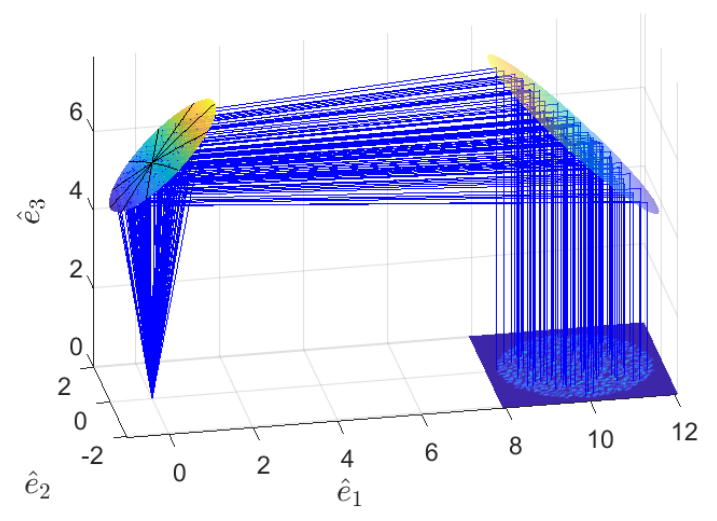

(c) $\varphi=\pi, \beta=22, w_{0}=6$.

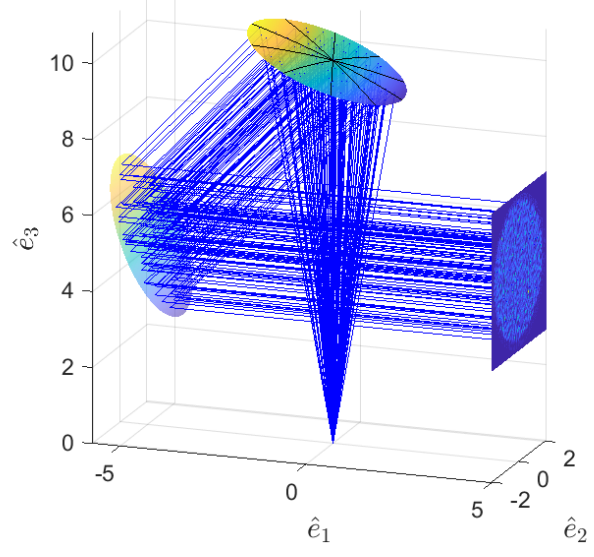

(b) $\varphi=\frac{\pi}{2}, \beta=22, u_{0}=10$.

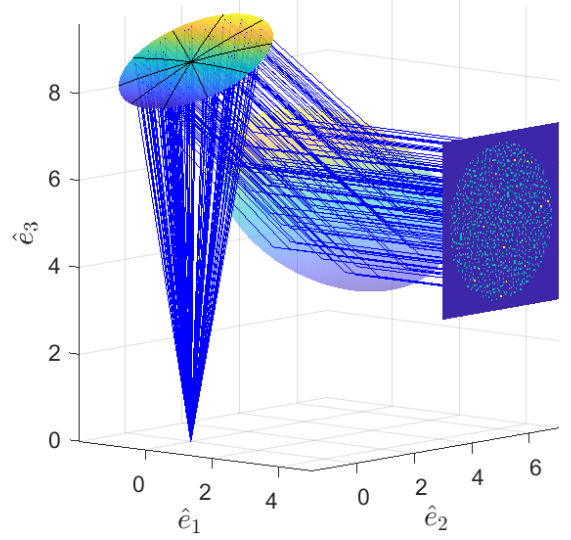

(d) $\varphi=\frac{\pi}{2}, \beta=15, w_{0}=5$.

Figure 2: Some examples of layouts constructed with our algorithm having a uniform source and target distribution.

ping criterion is met,

$$
\begin{aligned}
\boldsymbol{P}^{n+1} & =\underset{\boldsymbol{P} \in \mathcal{P}_{\left(\boldsymbol{m}^{n}\right)}}{\operatorname{argmin}} J_{\mathrm{I}}\left[\boldsymbol{m}^{n}, \boldsymbol{P}\right], \\
\boldsymbol{b}^{n+1} & =\underset{\boldsymbol{b} \in \mathcal{B}}{\operatorname{argmin}} J_{\mathrm{B}}\left[\boldsymbol{m}^{n}, \boldsymbol{b}\right], \\
\boldsymbol{m}^{n+1} & =\underset{\boldsymbol{m} \in \mathcal{M}}{\operatorname{argmin}} J\left[\boldsymbol{m}, \boldsymbol{P}^{n+1}, \boldsymbol{b}^{n+1}\right], \\
\boldsymbol{C}^{n+1} & =\boldsymbol{C}\left(\boldsymbol{x}, \boldsymbol{m}^{n+1}\right) .
\end{aligned}
$$

The first two steps can be solved pointwise, since they do not contain derivatives of $\boldsymbol{P}$ and $\boldsymbol{b}$, respectively. The minimizer for (15a) can be found analytically and exactly, as shown in [3]. The step (15b) is explained in [4].

The computation of the mapping is a bit more intricate, since the functional $J$ includes derivatives of $\boldsymbol{m}$. The minimization is done using calculus of variations [1]. By setting the first variation of $J$ with respect to any $\boldsymbol{\eta} \in \mathcal{M}$ equal to 0 , we can eventually derive the following bound- ary value problem:

$$
\begin{gathered}
\operatorname{div}\left(\boldsymbol{C}^{T} \boldsymbol{C D m}\right)=\operatorname{div}\left(\boldsymbol{C}^{T} \boldsymbol{P}\right), \quad \text { for } \boldsymbol{x} \in \mathcal{X}, \\
\alpha \boldsymbol{C}^{T} \boldsymbol{C}(\mathrm{D} \boldsymbol{m}) \hat{\boldsymbol{n}}+(1-\alpha) \boldsymbol{m}=\alpha \boldsymbol{C}^{T} \boldsymbol{P} \hat{\boldsymbol{n}}+(1-\alpha) \boldsymbol{b} \\
\text { for } \boldsymbol{x} \in \partial \mathcal{X},
\end{gathered}
$$

where div is the divergence operator applied to each row of a matrix and $\hat{\boldsymbol{n}}$ is the outward unit normal of $\mathcal{X}$. This system is discretized using a finite volume method and the resulting linear system is solved using a QR-decomposition. After convergence, we calculate the surface shapes from the mapping $\boldsymbol{m}$. This is done by using Eq. (7), finding a $u_{1}$ that minimizes

$$
I[\phi]=\frac{1}{2} \iint_{\mathcal{X}}\left|\nabla_{x} c(., \boldsymbol{m})-\nabla \phi\right|^{2} \mathrm{~d} \boldsymbol{x} .
$$

The surface shapes are then calculated from $u_{1}$ using Eq. (4). This calculation introduces an extra degree of freedom, which we use to define the position of one of the two 
surfaces along the central ray, i.e. $u_{0}$ or $w_{0}$.

In this design problem, there are some degrees of freedom in the choice of input parameters. Obviously, $f$ and $g$ are given as input, as well as the angles $\varphi$ and $\theta$ for the direction of the outgoing beam. However, for a rotationally symmetric $f$, the angle $\theta$ does not matter and can be chosen equal to 0 . Furthermore, two out of $L, l$ and $\beta$ are input parameters, and either $u_{0}$ or $w_{0}$.

\section{Numerical results}

We tested our method with two cases. First, we consider the case of a uniform source and target. This is used to show the possibilities of designing optical systems with different layouts. A common layout has the output beam parallel to the symmetry axis of the source output, see Fig. 2a. After a Monte Carlo ray trace with ten million rays we obtain a standard deviation of $4.4 \%$ of the mean flux in a bin. This simulation was done on a $200 \times 200$ grid in 200 iterations, taking 167 seconds. As mentioned, we can change the output direction. Since our source is rotationally symmetric, only the polar angle $\varphi$ of $\hat{\boldsymbol{a}}_{3}$ matters. By varying this angle, the reduced optical path length $\beta$ and the central position of the first or second mirror ( $u_{0}$ and $w_{0}$, respectively), we can construct optical systems such as in Fig. 2.

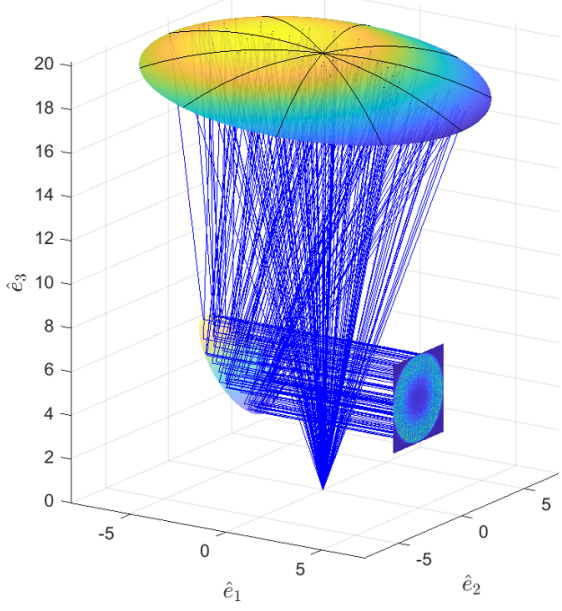

Figure 3: The layout of our test case using a laser diode and an annular output.

The second test case is a model of a laser diode source and a ring-shaped target. This specific laser beam output can be used in practical applications. The laser diode is modeled by an elliptical Gaussian intensity from the point source having $1 / e^{2}$ intensity angles $\theta_{x}=45^{\circ}$ and $\theta_{y}=13^{\circ}$. The ring-target has an inner radius of 1 and an outer radius of 2. The layout of the optical system is given in Fig. 3. The polar angle of the output direction is given by $\varphi=\pi / 2$. Furthermore, $\beta=30, l=5$, the distance of the first reflector along the central ray is 20 and the center of the ring is shifted by 5 along the $\hat{\boldsymbol{e}}_{3}$-axis.

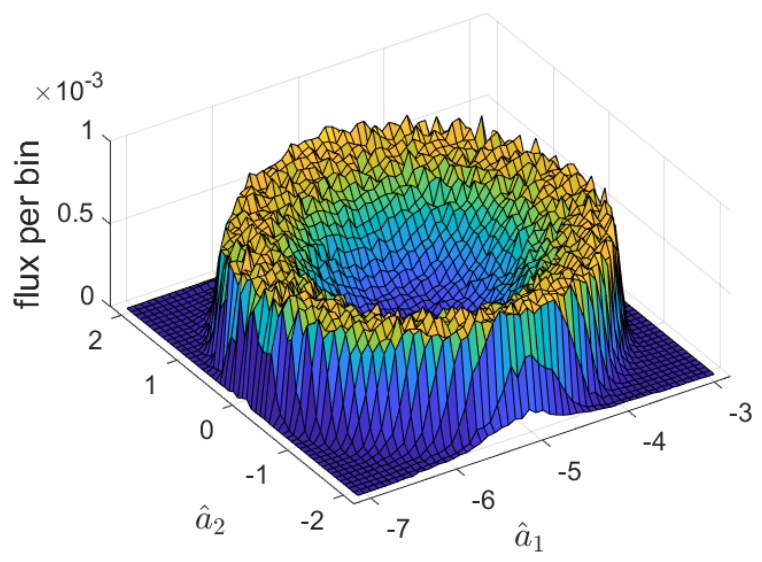

Figure 4: Raytraced resulting output illumination for the second test case

Using our algorithm to design an optical system and applying ray tracing gives an output illumination as in Fig. 4. This test case is more elaborate than the uniform case and therefore takes longer to converge. Using a $400 \times 400$ grid and 1000 iterations takes approximately $3000 \mathrm{sec}-$ onds.

\section{Conclusion}

To conclude, we presented a method to design tworeflector systems shaping and collimating light for a wide range of input and output distributions. Because the output direction can be chosen arbitrarily, there is a high flexibility in choosing the layout of the system.

\section{References}

[1] T.A.H. van Roosmalen, J.H.M. ten Thije Boonkkamp, M.J.H. Anthonissen, W.L. IJzerman, Optics Express 29 (2021), preprint arXiv:2104.04468

[2] C.R. Prins, R. Beltman, J.H.M. ten Thije Boonkkamp, W.L. IJzerman, T.W. Tukker, SIAM Journal on Scientific Computing 37, B937 (2015)

[3] N.K. Yadav, Ph.D. thesis, Eindhoven University of Technology (2018)

[4] L.B. Romijn, J.H.M. ten Thije Boonkkamp, W.L. IJzerman, Journal of Computational Physics 408 (2020)

[5] E. Hecht, Optics (Pearson, 2016), ISBN 9781292096933 